\title{
A Case of Meropenem Induced Cholestasis
}

\author{
Busra Can, Ozgur Kara, Gunes Arık, Gözde Sengul Aycicek, Fatih Sumer, Zekeriya Ulger \\ Department of Geriatrics, Gazi University, Ankara, Turkey
}

\begin{abstract}
Meropenem is a broad-spectrum carbapenem antibiotic used for complicated hospital infections. Mild aminotransferase elevations have been reported in meropenem recipients, but cholestasis is rare. A 70 year old man was admitted for infected diabetic foot ulcer. ALP and GGT levels started increasing one week after the initiation of meropenem, with normal ALT, AST and bilirubin levels. Meropenem induced intrahepatic cholestasis was suspected and meropenem was discontinued, followed by normalization of liver enzymes.
\end{abstract}

Drug induced liver injury should be suspected in patients with unexplained liver enzyme elevations. $J$ Microbiol Infect Dis 2016;6(4): 190-191

Keywords: Meropenem; drug induced liver injury; cholestasis

\section{INTRODUCTION}

Meropenem is a broad-spectrum carbapenem antibiotic with excellent activity against many grampositive and gram-negative aerobic, facultative, and anaerobic bacteria. It is widely used for complicated hospital infections. Mild aminotransferase elevations have been reported in meropenem recipients, but cholestasis is rare. We describe a patient who developed intrahepatic cholestasis after meropenem administration for the treatment of an infected diabetic foot ulcer. Verbal informed consent to publish patient information was obtained from the patient.

\section{CASE}

A 70-year-old man with type 2 diabetes mellitus, hypertension and coronary artery disease was admitted to the Geriatric Ward with diabetic foot infection. On physical examination his body temperature was $36.8^{\circ} \mathrm{C}$ with normal blood pressure and heart rate. He had a $5 \times 8 \mathrm{~cm}$ necrotic ulcer on his left heel, which was debrided upon admission. His second left toe was amputated a year ago and the stump appeared infected. Rest of the exam was unremarkable. The blood test results were as follows (with reference ranges): hemoglobin:10 g/dl (13-17); white blood cell count:14x103 cells/mm3 (4.5-11 x $\left.10^{3}\right)$; neutrophil count: $12 \times 10^{3}$ cells $/ \mathrm{mm}^{3}(1.9-8 \times$ $\left.10^{3}\right)$; platelets: $105 \times 103$ cells/mm3 (130-400x103); fasting glucose:180 mg/dL (74-106); creatinine:1.2 $\mathrm{mg} / \mathrm{dL}$ (0.7-1.2); blood urea nitrogen(BUN):24 mg/ dL (8-23); albumin: $2.9 \mathrm{~g} / \mathrm{dL}$ (3.5-5); alanine aminotransferase (ALT):14 U/L (5-40); aspartate aminotransferase (AST): $20 \mathrm{U} / \mathrm{L}$ (5-41); alkaline phosphatase (ALP):92 U/L (40-130); gamma glutamyltransferase (GGT): $22 \mathrm{U} / \mathrm{L}$ (0-55); lactate dehydrogenase (LDH): $226 \mathrm{U} / \mathrm{L}$ (135-225); total bilirubin: $0.18 \mathrm{mg} / \mathrm{dL}$ (0.1-1.4); C-reactive protein:134 mg/L (0-6); erythrocyte sedimentation rate: $94 \mathrm{~mm} / \mathrm{hr}(0-15)$. Serum electrolytes were normal and viral markers for hepatitis were negative. Plain radiograph of the foot showed no osteomyelitis. His medications included aspirin, carvedilol, ramipril and insulin glargine.

The wound swab demonstrated leucocytes, Gram positive cocci, Gram negative bacilli and Gram positive bacilli. Meropenem 500 mg every 8 hours was started after cultures were collected. E.coli was isolated from the initial tissue specimens. One week after the initiation of meropenem, ALP and GGT levels started to increase while ALT, AST and bilirubin levels remained normal (Table 1). Ultrasound of the abdomen revealed no biliary obstruction. Antimitochondrial antibody (AMA) test for primary biliary cirrhosis was negative. Abdominal MRI (magnetic resonance imaging) and MRCP (magnetic resonance cholangiopancreatography) 
ruled out primary sclerosing cholangitis and infiltrative diseases. Meropenem induced intrahepatic cholestasis was suspected and meropenem was discontinued. Naranjo adverse drug reaction probability scale indicated a probable relationship (score of 6 ) between the patient's cholestasis and meropenem therapy [1]. Liver enzymes fell into the normal range over the course of one month, which supported the diagnosis of "drug induced liver injury".

Table 1. The treatment course and associated laboratory tests

\begin{tabular}{ccccccc}
\hline Meropenem day & ALP & GGT & ALT & AST & LDH & T. Bil. \\
\hline 0 & 100 & 37 & 41 & 38 & 173 & 0.25 \\
\hline $7^{*}$ & 466 & 256 & 41 & 20 & 172 & 0.54 \\
10 & 337 & 187 & 28 & 12 & 207 & 0.34 \\
14 & 289 & 151 & 40 & 20 & 208 & 0.35 \\
40 & 130 & 69 & 16 & 23 & 225 & 0.38 \\
\hline
\end{tabular}

ALP: alkaline phosphatase (normal range: 40-130 U/L); GGT:gammaglutamyl transferase (normal range: 0-55 U/L); ALT:alanine aminotransferase (normal range: 5-40 $\mathrm{U} / \mathrm{L}$ ); AST:aspartate aminotransferase (normal range: 5-41 U/L); LDH:lactate dehydrogenase (normal range: 135-225 U/L); T. bil/total bilirubin (normal range: 0.1-1.4 $\mathrm{mg} / \mathrm{dL})$.

*Meropenem is discontinued

\section{DISCUSSION}

Drug-induced liver injury (DILI) is estimated to have a yearly incidence between $10-15$ per 10,000 100,000 [2]. In order to attribute liver injury to a drug, other liver diseases should be excluded; liver injury should be followed by drug exposure; and withdrawal of the culprit drug should lead to improvement [3] There are three types of DILI: hepatocellular (cytotoxic), cholestatic, and mixed. DILI cholestasis is defined as an elevated ALP $>2$ times the upper limit of normal and/or an ALT to ALP ratio of less than 2 [4]. Beta-lactam antibiotics are well recognized and studied causes of cholestasis. However, meropenem induced cholestasis is rarely reported.

Drug induced liver injury should be part of the differential diagnosis in patients with unexplained elevations in liver enzymes. The clinical presentation may be anywhere in the spectrum from asymptomatic disease to vanishing bile duct syndrome. Suspected drug/s should be immediately discontinued and the patient monitored closely.

Declaration of Conflicting Interests: The authors declare that they have no conflict of interest.

Financial Disclosure: No financial support was received.

\section{REFERENCES}

1. Naranjo CA, Busto U, Sellers EM, et al. A method for estimating the probability of adverse drug reactions. Clin Pharmacol Ther 1981;30:239-45

2. Holt M, Ju C. Drug-induced liver injury. Handb Exp Pharmacol 2010;3.

3. Navarro VJ, Senior JR. Drug-related hepatotoxicity. N Engl J Med 2006; 354:731

4. Bjornsson ES, Jonasson JG. Drug-induced cholestasis. Clin Liver Dis 2013; 17:191 